\title{
Biomedical Applications of Metal Additive Manufacturing: Current State-of-the-Art and Future Perspective
}

\author{
Shonak Bhattacharya ${ }^{1}$, Jenniffer Bustillos ${ }^{1}$, Fernando Quevedo Gonzalez ${ }^{2}$, Jason A Spector ${ }^{3,4}$, \\ and Atieh Moridi ${ }^{1 *}$ \\ ${ }^{1}$ Sibley School of Mechanical and Aerospace Engineering, Cornell University, USA \\ ${ }^{2}$ Department of Biomechanics, Hospital for Special Surgery, USA \\ ${ }^{3}$ Division of Plastic Surgery, Weill Cornell Medicine-New York Presbyterian Hospital, USA \\ ${ }^{4}$ Nancy E. and Peter C. Meining School of Bioengineering, Cornell University, USA
}

*Corresponding author: Atieh Moridi, Sibley School of Mechanical and Aerospace Engineering, Cornell University, Ithaca, NY, USA.

To Cite This Article: Atieh Moridi, Biomedical Applications of Metal Additive Manufacturing: Current State-of-the-Art and Future Perspective. Am J Biomed Sci \& Res. 2020 - 7(1). AJBSR.MS.ID.001103. DOI: 10.34297/AJBSR.2020.07.001103.

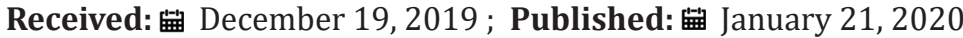

\begin{abstract}
Recent developments in Additive Manufacturing (AM) have provided unprecedented opportunities for emerging metal implant technologies. These technologies can combine unique architectures tailored to patient-specific needs, and potentially absorbable properties that could aid in the healing process. Advanced imaging techniques enable the reconstruction of the affected regions and the design of the implant, while AM can be used to print the patient-specific implant. Metallic metamaterials have been fabricated based on designed lattice structures with precisely controlled porous structures having specific functional and mechanical properties for the desired application. This review summarizes the current state-of-theart and future opportunities for metal AM in biomedical applications.
\end{abstract}

Keywords: Additive manufacturing; Patient-specific implants; Absorbable implants; Meta implants

\section{Introduction}

In the last decade, advancements in metal Additive Manufacturing (AM) have revolutionized the biomedical field by providing an innovative asset for the development of new clinical technologies (e.g. guides for surgical procedures, scaffolds for tissue engineering, and load bearing implants) [1-6]. The majority of metal AM technologies rely on the continuous fusion of a metallic feedstock (powder, wire or sheets) using a high energy source (laser, electron beam or plasma) in a layer-by-layer fashion to manufacture structures based on a user-defined computer aided design (CAD) model [4,7]. Based on its intrinsic layer-by-layer operation, AM allows the fabrication of structures with complex geometries that cannot be achieved by conventional subtractive methods $[2,3,8]$. The ease of design customization and the achievable structural complexity via AM could present a new solution to unanswered challenges in orthopedics [2-4,8-10]. These challenges include the inability for current metallic implants to mimic the structure and mechanical properties of the bone, which in turn can compromise the fixation of orthopedic implants, inaccurate anatomical fitting that adversely affect surgical placement of the devices, and limited restoration of the biological function of the remaining bone surrounding the implant [11-14].

The versatility and high resolution achieved by various AM techniques provides opportunities to engineer implants with hierarchical microstructures and tailored mechanical properties unique to each anatomic application [2,3]. Furthermore, the implants can be made patient-specific and designed to achieve desired biomechanical responses to aid in the restoration of physiological functions, such as controlled displacements for respiratory expansion and contraction [11]. Among metal AM processes, Selective Laser Melting (SLM) [15], and Electron Beam 
Melting (EBM) [16] are the most widely used methods for the fabrication of zygomatic bone [17], finger [18], knee [19], hip [20], craniofacial [21-24], and thoracic implants [12,14]; with several devices having received United States Food and Drug Administration (FDA) clearance [25].

This review focuses on the advantages and significant contributions of AM to modern orthopedics. The fabrication of metallic metamaterials with osteoconductive properties and tailored mechanical/functional responses to cater to patientspecific needs are discussed. Moreover, the prospect to develop novel absorbable implants using alloys of biodegradable metals is also reviewed.

\section{Recent Advances in AM for Orthopedic Implants}

\section{Topology optimization}

Topology Optimization is a promising tool to discover novel architectures with desirable structural and functional properties. This optimization method seeks to determine the optimal material distribution that will fulfils a set of pre-defined boundary conditions [26-28]. The optimized structures usually have complex geometries that are difficult to manufacture using conventional methods but can be produced owing to the geometric freedom offered by AM. Topology optimization is a promising route to discover novel architectures with desirable structural and functional properties $[27,29,30]$.

In addition to optimizing based on mechanical constraints, topology optimization can integrate fluidic boundary conditions that will account for the required permeability for tissue ingrowth maximizing cell migration and mass transport $[30,31]$. This aspect of topology optimization has been used outside of orthopedics, in developing efficient metallic stents for treating aneurisms, and in making scaffolds for regeneration of tissues $[32,33]$.

\section{Meta-implants}

The mechanical properties of conventional materials are primarily dictated by their chemical composition and their microstructure. Mechanical metamaterials challenge these conventional material definitions by engineering structures that exhibit counter-intuitive mechanical properties (e.g. materials that display remarkably low stiffness or a zero or negative Poisson's ratio). Metamaterials are often characterized by having ordered cellular lattices relating their density to their mechanical response and functionality and thus allowing them to be controlled. In the fabrication of orthopedic implants, metamaterials can provide structures with gradient porosities and stiffness similar to the intricate structure of bone in which cortical bone with high modulus (13.6GPa-28GPa) morphs regionally into low modulus cancellous bone (0.02GPa-0.64GPa) [34].

Complex metamaterial structures could serve to improve the interactions at the bone-implant interface and exhibit osteoconductive properties providing an optimized environment for tissue integration [10,35]. Meta-implants manufactured using tetrahedral unit cells and customized gradient densities have shown potential in reducing bone-loss resulting from stress shielding by $\sim 75 \%$ [36]. Orthopedic implants often incorporate intramedullary stems for fixation to the endosteal surfaces of long bones like the femur and humerus. The resulting implant bone composite is constantly subjected to bending and torsional loads, often leading to failure of the bone-implant interface. Moreover debris (caused by fretting wear) can get trapped at the interface causing osteolysis [37].

The design of implants combining auxetic and non-auxetic metamaterials on the interfaces subject to tensile and compressive loading respectively, have shown the ability to eliminate the retraction of the implant from the bone by inducing expansion at both interfaces, even as the material undergoes tensile loading [38]. This can result in increased implant longevity and reduced need for revision surgery.

\section{Patient-specific implants}

Despite the widespread availability of implant systems with several sizes to fit a broad spectrum of anatomy, cases nonetheless arise in which a standard commercial device will not suffice. Examples include cases of dysplasia where the anatomy is grossly distorted or cases of revision surgery in which the failure of the original implant has led to significant bone loss. Developing solutions for such cases can be reached by the direct coupling of advanced imaging techniques, usually Computed Tomography (CT) and Magnetic Resonance Imaging (MRI), with AM processes to engineer patient-specific implants. In addition, the same imaging techniques could be used to provide quality control for AM manufactured implants. CT data has the added benefit of providing direct high-resolution measurement of the density of the remaining bone.

This information can be used to help design not just the shape of the implant, but it's local structural and mechanical properties. In this way load transfer between the implant and the surrounding remnant bone can be optimized to assure both short-term fixation and longevity $[39,40]$. The direct reconstruction of anatomical regions via CT images has been effectively implemented for the accurate design of orbital socket [41], hip [42], and sternal implants [12] manufactured via AM techniques. The surgical procedures with sternal implants demonstrated immediate structural support and significant improvement in patients' respiratory symptoms post-operatively [12].

\section{Absorbable metallic implants}

Titanium alloys [43], cobalt-chromium alloys [44], tantalum and stainless steel $[45,46]$ are metallic materials commonly used in permanent orthopedic implants due to their high structural integrity, corrosion resistance and biocompatibility. However, 
numerous applications exist in which a lack of permanence is desired. For example, metallic plates and screws are often used to 'rigidly fix' fractured bones to facilitate healing. Once the fracture is healed, plates and screws can become a source of infection and stress shielding, and at times a cause of discomfort for the patient. Moreover, in pediatric patients, metal implants must often be removed to allow bone growth as the child gets older. The process of removing the implant requires another surgery, which comes with an additional risk of surgical complications, pain and rehabilitation and of course, additional financial burden.

Although, polymers have long been used to make resorbable implants, they do not meet the structural requirements needed in plates, screws or most load bearing implants. In addition, degradation of synthetic polymer-based resorbable structures have shown to result in inflammation and the formation of 'sterile' abscesses [47]. Magnesium, iron and zinc are metals with bioresorbable properties and are essential trace metals in normal human physiology [48]. Using alloys of these metals, implants can be manufactured with AM to have tuneable degradation rates that match the rate of bone formation for the given application reducing the risk of infection and cytotoxicity [49,50]. Further, these implants could release metal ions in non-toxic quantities that may even be beneficial to the healing process [51].

\section{Conclusions and Future Perspectives}

AM of metals in biomedical applications has seen a steady increase in interest over the past 10 years. According to the US National Library of Medicine (PubMed), the number of publications (peer reviewed articles, case reports, and clinical trials) in this field have increased from 4,500 in 2010, to approximately 9,500 in 2019. The rapid growth of AM has equipped us with the capability to manufacture long-lasting implants with greater structural compatibility with the bone. AM processed implants have been effectively incorporated into surgical practice in recent years. The development of engineered metallic metamaterials with functional mechanical responses promises to address some of the limitations of conventionally manufactured implants in terms of their geometry, density, strength and stiffness.

The application of CT imaging to reconstruct the affected region andfabricatepatient-specificimplantshas resultedinunprecedented progress in reconstructive implants. Another benefit is the use of absorbable implants to provide a temporary scaffold to support the bone healing without permanently remaining in the body. Despite great progress in adopting AM for biomedical applications, certain areas require further improvement to develop the full potential of this disruptive manufacturing technology. Although the use of topology optimization is growing, we are still far from the largescale adoption of this computational tool. In addition, improving metal AM resolution from microscale to nanoscale is required to advance the capability of printing complex structures. Improving geometrical fidelity could also result in greater implementation of topology optimization methods as we start to print smaller features with higher precision. However, an unsurmountable trade-off exists between deposition rate and accuracy that will remain a guiding limitation in the design of new processes with enhanced resolution.

Similarly, the optimization of stiffness to enhance load sharing and load transfer between implant and bone cannot be done without consideration of fatigue, as orthopedic implants are subjected to continual cyclic loading. This becomes a more complicated problem as we print materials with ever more complex geometries. Understanding the deformation mechanisms and the effects of feature size/length scale and interconnectivity on mechanical and degradation properties of implants is important to ensure proper implant design. To overcome these challenges, interdisciplinary collaborations between engineers and clinicians is a crucial step toward medical discoveries and their clinical translation to treat patients.

Scientific knowledge and engineering limitations are not the only factors currently hampering the widespread use of AM in orthopedic devices. Necessary regulation by the FDA to ensure the safety and efficacy of orthopedic implants is an important factor when adopting AM into the manufacturing of both standard and patient-specific implants. Areas that must be considered as part of the regulation of AM manufactured implants include: the starting materials; design, printing and post-printing validation of the AM process; printing characteristics and parameters; physical and mechanical assessment of the final implants; and biological considerations of these implants, including cleaning, sterility and biocompatibility. The FDA has been working with the commercial, clinical and scientific communities to provide guidance on AM in medical devices.

\section{Acknowledgment}

Authors would like to thank Dr. Timothy Wright for the helpful discussion and suggestions.

\section{Conflict of Interest}

The authors declare no conflict of interest.

\section{References}

1. Yang Y, Wang G, Liang H, Gao C, Peng S, et al. (2019) Additive manufacturing of bone scaffolds. Int J Bioprinting 5(1): 1-25.

2. Warnke PH, Douglas T, Wollny P, Sherry E, Steiner M, et al. (2009) Rapid prototyping: Porous titanium alloy scaffolds produced by selective laser melting for bone tissue engineering. Tissue Eng Part C Methods 15(2): 115-124.

3. Van der Stok J, Van der Jagt OP, Amin Yavari S, De Haas MFP, Waarsing JH, et al. (2013) Selective laser melting-produced porous titanium scaffolds regenerate bone in critical size cortical bone defects. J Orthop Res 31(5): 792-799.

4. Frazier WE (2014) Metal Additive Manufacturing: A Review. J Mater Eng Perform 23(6): 1917-1928.

5. Das A, Moridi A (2019) State of the art in Directed Energy Deposition: From Additive Manufacturing to Materials Design. Coatings 9(7): 418. 
6. Mahmoud D, Elbestawi M (2017) Lattice Structures and Functionally Graded Materials Applications in Additive Manufacturing of Orthopedic Implants: A Review. J Manuf Mater Process 1(2): 13.

7. Regenfuss P, Streek A, Hartwig L, Klötzer S, Brabant T, et al. (2007) Principles of laser micro sintering. Rapid Prototyp J 13(4): 204-212.

8. de Jonge, CP, Kolken HMA, Zadpoor AA (2019) Non-auxetic mechanical metamaterials. Materials 12(4) 635.

9. Winkler T, Sass FA, Duda GN, Schmidt-Bleek K (2018) A review of biomaterials in bone defect healing, remaining shortcomings and future opportunities for bone tissue engineering: The unsolved challenge. Bone Jt Res 7(3): 232-243.

10. Heinl P, Müller L, Körner C, Singer RF, Müller FA (2008) Cellular Ti-6Al$4 \mathrm{~V}$ structures with interconnected macro porosity for bone implants fabricated by selective electron beam melting. Acta Biomater 4(5): 1536-1544.

11. Moradiellos J, Amor S, Córdoba M, Rocco G, Vidal M, et al. (2017) Functional Chest Wall Reconstruction with a Biomechanical ThreeDimensionally Printed Implant. Ann Thorac Surg 103(4): 389-391.

12. Kamel MKK, Cheng A, Vaughan B, Stiles B, Altorki N, et al. (2019) Sternal Reconstruction Using Customized 3D-Printed Titanium Implants. Ann Thorac Surg 4975(19): 31720-31725.

13. Aragón J, Méndez IP (2016) Dynamic 3D printed titanium copy prosthesis: A novel design for large chest wall resection and reconstruction. J Thorac Dis 8(6): 385-389.

14. Aranda JL, Jiménez MF, Rodríguez M, Varela G (2015) Tridimensional titanium-printed custom-made prosthesis for sternocostal reconstruction. Eur J Cardio Thoracic Surg 48(4): 92-94.

15. Moridi A, Demir AG, Caprio L, Hart AJ, Previtali B, et al. (2019) Deformation and failure mechanisms of Ti-6Al-4V as built by selective laser melting. Mater Sci Eng A 768: 138456.

16. Ataee A, Li Y, Song G, Wen C (2017) Metal scaffolds processed by electron beam melting for biomedical applications. In Met Foam Bone Process. Modif Charact Prop pp.83-110.

17. Rotaru H, Schumacher R, Kim SG, Dinu C (2015) Selective laser melted titanium implants: a new technique for the reconstruction of extensive zygomatic complex defects. Maxillofac Plast Reconstr Surg 37(1): 1.

18. Merkt S, Kleyer A, Hueber AJ (2014) The Additive Manufacture of Patient-tailored Finger Implants. Laser Tech J 11(2): 54-56.

19. Murr LE, Amato KN, Li SJ, Tian YX, Cheng, et.al. (2011) Microstructure and mechanical properties of open-cellular biomaterials prototypes for total knee replacement implants fabricated by electron beam melting. J Mech Behav Biomed Mater 4(7): 1396-1411.

20. Cronskär M, Bäckström M, Rännar LE (2013) Production of customized hip stem prostheses-A comparison between conventional machining and electron beam melting (EBM). Rapid Prototyp J 19(5): 365-372.

21. Jardini AL, Larosa MA, de Carvalho Zavaglia CA, Bernardes LF, Lambert CS, et al. (2014) Customized titanium implant fabricated in additive manufacturing for craniomaxillofacial surgery. Virtual Phys Prototyp 9(2): 115-125.

22. Jardini AL, Larosa MA, Filho RM, de Carvalho Zavaglia, Bernardes LF, et al. (2014) Cranial reconstruction: 3D biomodel and custom-built implant created using additive manufacturing. J Cranio-Maxillofacial Surg 42(8): 1877-1884.

23. Mazzoli A, Germani M, Raffaeli R (2009) Direct fabrication through electron beam melting technology of custom cranial implants designed in a PHANToM-based haptic environment. Mater Des 30(8): 3186-3192.

24. Sutradhar A, Paulino GH, Miller MJ, Nguyen TH (2010) Topological optimization for designing patient-specific large craniofacial segmental bone replacements. Proc Natl Acad Sci USA 107(30): 13222-13227.
25. Sing SL, An J, Yeong WY, Wiria FE (2016) Laser and electron-beam powder-bed additive manufacturing of metallic implants: A review on processes, materials and designs. J Orthop Res 34(3): 369-385.

26. Challis VJ, Guest JK (2009) Level set topology optimization of fluids in Stokes flow. Int J Numer Methods Eng 79(10): 1284-1308.

27. Lin CY, Kikuchi N, Hollister SJ (2004) A novel method for biomaterial scaffold internal architecture design to match bone elastic properties with desired porosity. J Biomech 37(5): 623-636.

28. Dbouk T (2017) A review about the engineering design of optimal heat transfer systems using topology optimization. Appl Therm Eng 112: 841-854.

29. Kang H, Long JP, Goldner GDU, Goldstein SA, Hollister SJ (2012) A paradigm for the development and evaluation of novel implant topologies for bone fixation: Implant design and fabrication. J Biomech 45(13): 2241-2247.

30. Hollister SJ (2005) Porous scaffold design for tissue engineering. Nat. Mater. Nature Publishing Group 4: 518-524.

31. Hyun J, Wang S, Yang S (2014) Topology optimization of the shear thinning non-Newtonian fluidic systems for minimizing wall shear stress. Comput Math with Appl 67(5): 1154-1170.

32. Jiang L, Chen S, Sadasivan C, Jiao X (2017) Structural topology optimization for generative design of personalized aneurysm implants: Design, additive manufacturing, and experimental validation. 2017 IEEE Healthc. Innov Point Care Technol pp.9-13.

33. Challis VJ, Roberts AP, Grotowski JF, Zhang LC, Sercombe TB (2010) Prototypes for Bone Implant Scaffolds Designed via Topology Optimization and Manufactured by Solid Freeform Fabrication. Adv Eng Mater 12(11): 1106-1110.

34. Zhang L, Chen L (2019) A Review on Biomedical Titanium Alloys: Recent Progress and Prospect. Adv Eng Mater 21(4): 1801215.

35. Schaedler TA, Jacobsen AJ, Torrents A, Sorensen AE, Lian J, et al (2011) Ultralight metallic microlattices. Science 334(6058): 962-965.

36. Arabnejad S, Johnston B, Tanzer M, Pasini D (2017) Fully porous 3D printed titanium femoral stem to reduce stress-shielding following total hip arthroplasty. J Orthop Res 35(8): 1774-1783.

37. Macinnes SJ (2016) The Genetics of Osteolysis and Heterotopic Ossification After Total Hip Arthroplasty pp.241.

38. Kolken HMA, Janbaz S, Leeflang SMA, Lietaert K, Weinans HH, et al. (2018) Rationally designed meta-implants: A combination of auxetic and conventional meta-biomaterials. Mater Horizons 5(1): 28-35.

39. Celenk C, Celenk P (2012) Bone Density Measurement Using Computed Tomography. Comput Tomogr Clin Appl pp.16.

40. Bruno V, Berti C, Barausse C, Badino M, Gasparro R, et al. (2018) Clinical Relevance of Bone Density Values from CT Related to Dental Implant Stability: A Retrospective Study. Biomed Res Int pp. 8.

41. Stoor P, Suomalainen A, Lindqvist C, Mesimäki K, Danielsson, et al. (2014) Rapid prototyped patient specific implants for reconstruction of orbital wall defects. J Cranio Maxillofacial Surg 42(8): 1644-1649.

42. Jun Y, Choi K (2010) Design of patient-specific hip implants based on the 3D geometry of the human femur. Adv Eng Softw 41(4): 537-547.

43. Hao YL, Li SJ, Yang R (2016) Biomedical titanium alloys and their additive manufacturing. Rare Met 35(9): 661-671.

44. Limmahakhun S, Oloyede A, Sitthiseripratip K, Xiao Y, Yan C (2017) Stiffness and strength tailoring of cobalt chromium graded cellular structures for stress-shielding reduction. Mater Des 114: 633-641.

45. Santos PF, Niinomi M, Liu H, Cho K, Nakai, et al. (2016) Fabrication of low-cost beta-type Ti-Mn alloys for biomedical applications by metal injection molding process and their mechanical properties. J Mech Behav Biomed Mater 59: 497-507. 
46. Karamian E, Motamedi MRK, Khandan A, Soltani P, Maghsoudi S (2014) An in vitro evaluation of novel NHA/zircon plasma coating on 316L stainless steel dental implant. Prog Nat Sci Mater Int 24(2): 150-156.

47. Sheikh Z, Najeeb S, Khurshid Z, Verma V, Rashid H, et al. (2015) Biodegradable materials for bone repair and tissue engineering applications. Materials 8(9): 5744-5794.

48. Hermawan H (2018) Updates on the research and development of absorbable metals for biomedical applications. Prog Biomater 7(2): 93110.
49. Liu B, Zheng YF (2011) Effects of alloying elements (Mn, Co, Al, W, Sn, B, $\mathrm{C}$ and $\mathrm{S}$ ) on biodegradability and in vitro biocompatibility of pure iron. Acta Biomater 7(3): 1407-1420.

50. Persaud-Sharma D, Mcgoron A (2012) Biodegradable magnesium alloys: A review of material development and applications. J Biomim Biomater Tissue Eng 12(1): 25-39.

51. U S Food and Drug Administration (2019) Biological Responses to Metal Implants. pp. 149. 